\title{
Intoxicaciones vegetales en la infancia
}

DR. WALDO BRUNING SCH, *

\section{I.- Conceptos Generales}

Dada la amplitud del tema, hemos creído conveniente diviđir su exposición en dos capítulos: uno dedicado a conceptos generales sobre toxicología vegetal, en relación con la pediatria, que es el que sigue a continuación y el otro, que será publicado en un próximo número, en que se consigna la nómina de nuestras principales plantas venenosas y sus características botánicas y toxoclínicas.

La permanente expansión de conocimientos en la ciencia botánica se ha manifestado en la creciente importancia de especialidades o ramas tales como la genética vegetal, la anatomía botánica, la ecología de las plantas y la fitopaleontología. El número de especializaciones sigue cn paulatino aumento y los progresos logrados en el conjunto de estudios botánicos durante el presente siglo han tenido trascendentales repercusiones en el nivel de vida de la moderna sociedad humana.

La toxicologia vegetal no podía mantenerse al margen de este avance, sobre todo después del impactante auge que experimentó en todo el orbe la disciplina toxicológica, en los últimos 25 años, y así también ella ha llegado a constituir otra inportante rama más, no sólo de la ciencia botánica, sino también de la clínica médica.

Las plantas tóxicas existentes en nuestro medio son por lo general nativas y silvestres, pero las hay también que son exóticas, en su mayoría orramentales, que crecen en jardines, invernaderos o interiores de las habitaciones. Las silvestres son habitualmente malezas, que se desarrolian en terrenos de barbecho, jardines o a lo largo de los

\footnotetext{
* Miembra del Comite de Redacción de la Revista Chilena de Pediatria.
}

caminos, en praderas o bosques. Otras son cordilleranas, algunas crecen en desperdicios o estiércol, en la vecindad de viviendas humanas y finalmente, unas pocas, vegetan en terrenos pantanosos.

Los principios activos, por medio de los cuales las plantas ejercen sus efectos tóxicos, están constituidos por alcaloides, glucósidos, aceites volátiles, ciertas sustancias indiferentes y algunas toxinas.

Los alcaloides son sustancias nitrogenadas complejas, de tipo básico, que se encuentran al estado soluble en el jugo celular o lechoso, como producto del netabolismo de numerosas especies. Forman con los ácidos, sales características de variada acción fisiológica, que se encuentran repartidas y combinadas de manera muy diversa en los difcrentes órganos vegetales.

Los glucósidos comprenden un numeroso grupo de sustancias químicas, de estructura también compleja, que se desintegran durante la fermentación o al ser tratados con ácidos minerales diluídes, dando lugar a azúcar y a ciertos compuestos vencnosos. El efecto tóxico se produce cuando los glucósidos y los fermentos o enzimas se ponen en contacto en el interior o exterior del organismo. Los de mayor importancia son los denominados cianogenéticos, que producen al descomponerse, ácido prúsico o cianhídrico (HCN) y los cardía$\cos$, como la digitalina y la estrofantina. Otras sustancias glucosídicas bastante difundidas, son las saponinas (sapotoxjnas), de reconocida acción hemolítica.

Muchas plantas contienen aceites volátiles o sustancias indiferentes, a menudo en combinación con otros tóxicos. Estos le confieren un olor o sabor desagradable y pungente, que induce generalmente a los niños a rehusar su ingestión. Ejer- 
cen su efecto por irritación y a veces coagulación proteínica y se pueden incorporar al torrente sanguíneo, causando daños en cualquier parte del organismo.

Finalmente, en algunas especics se encuentran ciertas toxinas (fitotoxinas), que no contienen nitrógeno (csto las diferencia de los alcaloides), se parecen a las enzimas en sus propiedades biológicas y producen un verdadero estado de inmunidad, mediante la formación de anticuerpos especificos o antitoxinas (antirricina, antirobina).

El grado de toxicidad de los vegetales depende de diversos factores inherentes tanto a ellos, como al medio ambiente en que se desarrollan. Entre estos factores corresponde señalar: el estado y la fase de crecimiento de las plantas, la composición y el grado de humedad del suelo, los abonos aplicados, las condiciones climáticas, la altitud y la influencia de los herbicidas hormonales. Rebasaría por cierto el ámbito de cste trabajo, si entráramos en mayores detalles al respecto.

Los tóxicos no están uniformemente distribuidos en todos los órganos de las plantas; a menudo están contenidos en todas sus partes, pero muchas especies forman la sustancia activa en una sola o en varias de ellas, manteniéndose inocuas las restantes. En algunas plantas son las raíces o tubérculos, o la cortcza, los principales reservorios de estas sustancias; en otras, se concentran en tallos y hojas y a veces es el fruto o la scmilla la parte más tóxica.

Aun cuando a primera vista se pudiera suponer, por la amplia difusión que alcanza el Reino Vegetal en casi todos los ámbitos del globo terráqueo, que las posibilidades de envenenamientos por plantas fueran ilimitadas, la realidad es muy otra y estos cuadros constituyen al menos en las regiones de clima templado y frío un rubro muy minoritario de la estadística general toxicológica.

Así en nuestro país se ha registrado una muy baja incidencia de cuadros fitotóxicos $(1,7$ a $2,1 \%$ ) (1), en la República Argentina su frecuencia es ligeramente mayor $(2,35 \%)$ (2) y sólo en Estados Unidos y Europa sư número es algo más elevado ( 4 a $8 \%$ ), lo que podría atribuirse a una cifra más alta de casos diagnosticados y probablemente también a un mayor consumo de setas. Así p. ej. Moeschlin refiere que entre las intoxicaciones de observación más frecuente en Europa, se cuentan las causadas por ingestión de hongos, con un 4\% (3). Ahora, en países de frondosa vegetación tropical se registra un mayor número de intoxicaciones que en aquellos de clima templado o frío. En abono de este hecho puede citarse una información proporcionada por Schvartsman, de Sao Paulo, según la cual, entre un 15,6 y un $24,5 \%$ de los niños hospitalizados en dicha ciudad, habría padecido cuadros fitotóxicos (4).
La causa de esta baja incidencia en nuestro país radica en el número relativamente reducido de especies tóxicas (de las 5.500 especies que aproximadamente existen, sólo unas 150 son reputadas como venenosas) y sobre todo en la repelencia de la mayoría de las plantas productoras de venenos, cuyo sabor u olor son por lo general notoriamente repugnantes, lo que determina su franco rechazo por los niños. Un ejemplo bien demostrativo en este sentido, nos lo proporciona la digital, la especic venenosa tal vez más generalizada y cuyo sabor es muy desagradable: en la literatura de los últimes 20 años no se ha registrado un solo caso de envenewamiento humano, por consumo de sus hojas verdes.

Los cuadros fitotóxicos son en la actualidad casi todos accidentales. En la Antigüedad y Epoca Medieval, en cambio, prevalecieron los de carácter delictivo, como nconteció por lo demás con todas las otras sustancias venenosas.

Así como desde el punto de vista toxicológica general, por lo menos un $70 \%$ de los casos accjdentales corresponde a la esfera pediátrica, también la gran mayoría de las intoxicaciones vegetales ocurre en niños. Como explicación de este hecho merece consignarse que un adulto, en su sano juicio, dificilmente ingiere partes $\mathbf{u}$ órganos vegetales no comestibles, al estado crudo. Además se debe considerar como circunstancia agravante que la mayor parte de los venenos actúa en los niños más intensa y drásticamente que en el adulto.

Algunos datos estadísticos pueden ilustrar sobre el particular. En una investigación realizada en las Postas de Urgencia de Santiago (1961-64) no se registró ningún caso de intoxicación en adultos, y en cambio sí 170 en niños (5); otra estadística emanada del Centro Suizo de Información Toxicológica (1966-68) en que fueron procesados 12.000 casos, arrojó s6lo 24 intoxicaciones vegetales en adultos y 476 en niños (6).

Tal como ocurre en envenenamientos motivados por otros agentes, son afectados prioritariamente niños de 1 a 5 años, vale decir, de una edad que con buen fundarnento puede denominarse "crítica", en que el pequeño es más susceptible a la acción tóxica por sus características psicológicas: su gran movilidad, su curiosidad y espíritu de imitación, su avidez por nuevos contactos y su apetito insaciable, y muchas veces perverso, que lo induce a ingerir las sustancias más extravagantes, lo cual tiene, como es obvio, particular vigencia en el caso de las intoxicaciones vegetales. Adcmás, por su temperamento inquieto y aventurero, los niños de sexo masculíno se exponen más fácilmente a este tipo de accidentes.

Aun cuando no son comparativas, se consignan a continuación irdagaciones estadísticas practicadas por Schvartsman, de Sao Paulo (7) y el 
National Clearinghouse for Poison Control Centers, de Washington (8).

De 327 intoxicaciones en niños de hasta 11 años, que se presentaron durante un lapso de 6 años (1963 a 1969) en el Hospital das Clinicas, de Sao Paulo, 8 correspondieron a niños de 1 a 2 an̆os; 38 a niños de 2 a 3 años; 63 a niños de 3 a 4 años y 69 a niños de 4 a 5 años. No se registraron cuadros tóxicos en menores de 1 año. La mayor incidencia se presentó en niños de 3 a 5 años.

El National Clearinghouse for Poison Control Centers, por su parte, investigó también sobre la frecuencia de los cuadros ocurridos en menores de 5 años, durante el año 1967, tomando como base una casuística de 2.884 envenenamientos registrados en 395 Centros de Información Toxicológica, de Estados Unidos. Se llegó a los siguientes resultados:

\begin{tabular}{lcr}
\hline Edad & Casas & $\%$ \\
\hline Menores de 1 año & 244 & 8,5 \\
1 a 2 años & 752 & 26,1 \\
2 a 3 años & 918 & 31,8 \\
3 a 4 años & 639 & 22,2 \\
4 a 5 años & 331 & 11,5 \\
\hline
\end{tabular}

El cuadro preinserto revela que el porcentaje más alto, $31,8 \%$, correspondió a niños de 2 a 3 años, en seguida $26,1 \%$ a niños de 1 a 2 años y después, en orden decreciente, $22,2 \%$ de 3 a 4 años, $11,5 \%$, de 4 a 5 años y por úlitimo, $8,5 \%$ a menores de 1 año.

En Chile no contamos con información sobre distribución etaria de Ias intoxicaciones vegetales.

A pesar de las características psicológicas del niño menor que acabamos de señalar, muchas intoxicaciones no llegan afortunadamente a consumarse, por el sabor tan desagradable u olor nauseabundo de la mayor parte de las plantas venenosas. Esto hace que los niños, aun cuando lleguen a mordisquear sus hojas o tallos, los escupan de inmediato, sin dar tiempo a que originen algún trastorno; no sucede to mismo con muchos frutos y semillas, cuyo bello colorido suele ejercer una gran atracción sobre ellos, facilitando de ese modo la ocurrencia de más de alguna intoxicación. De allí que exista manifiesta conveniencia en preservar a los niños del peligro que los acecha permaneutemente, de caer víctimas de una intoxicación vegetal, mediante una educación consecuente, instruyéndolos precozmente, desde la edad más temprana posible, sobre las principales características y el reconocimiento de las plantas vemenosas y acerca del riesgo a que se exponen cuando consumen sus frutos o demás organos. Se deberá insistir, con verdadera majadería, una y otra vez, sobre la absoluta prohibición de echarse a la boca cualquier parte de una planta que no conozcan bien - puedan identificar como un ejemplar inocuo. Sólo así porá evitarse más de algún envenenamiento o deceso jmputable a la desidia y despreocupación de los mayores.

En seguida pasaremos revista a los diversos mecanismos que intervienen en la gestación de las intoxicaciones vegetales. Estos deben diferenciarse según se produzcan:

1) en plantas que son tóxjcas por sí mismas;

2) en plantas alimenticias que se tornan tóxicas por un consumo excesivo o en ciertas fases de su crecimiento, almacenamiento o preparación;

3) en plantas contaminadas por causas ambientales;

4) en alimentos contaminados con sustancias tóxicas de procedencia vegetal, $y$

5 ) en niños con susceptibilidad individual (alergia tóxica).

Los envenenamientos que se originan por ingestión accidental de un órgano o parte de una planta, que es tóxica por sí misma, comprenden el grupo más numeroso y representativo de la toxicologia vegetal.

Ellos afectan de preferencia a aquellos niños, que, pese al sabor repelente de la mayoría de las especies tóxicas, consumen alguna de sus partes, (hojas, tallos, raíces, frutos o semillas) por mera curiosidad o hambre, o bien atraídos por su vistoso colorido.

Sobre todo los niños pequeños están expuestos a esta contingencia, por lo que se impone preventivamente ejercitar la mayor vigilancia sobre sus movimientos al aire libre e impartirles, de acuerdo a su edad, nociones sobre toxicidad de las plantas y las limitaciones que deberán observar, para coger sólo partes vegetales inofensivas.

En algunas oportunidades se pueden originar cuadros tóxicos, cuando niños ya mayores confunden especies venenosas con otras inocuas o comestibles, de características similares, como ser raíces de ranúnculos con chufas, hojas de cicuta con perejil, hojas de digital con borraja, frutas de dafne con grosellas, o de deu con moras.

Durante sus juegos y entretenimientos, los niños también pueden sufrir envenenamientos, por manipulación con ciertos órganos vegetales. Estos accidentes se suelen observar en contadas ocasiones y en ellos se produce más bien un contacto bucal prolongado o succión, preferentemente de tallos, 
ramas verdes o inflorescencias. Los niños están especialmente expuestos a sufrir este tipo de intoxicación cuando confeccionan "pitos" o "ffautas" con tallos huecos, o bien, cuando cortan ganchos verdes con los dientes. Cuadros de particular gravedad han sido descritos en niños que han preparado "flautas" con el tallo del arbusto denominado Deu o Coriaria (Coriaria myrtifolia L.).

En raras ocasiones, niños malcriados pueden por simple capricho o maldad contravenir expresas instrucciones de sus mayores y exteriorizar un persistente afán morboso por ingerir o succionar un determinado órgano vegetal; asimismo han sido descritos casos aislados de habituación a ciertas plantas tóxicas, en que algunos niños con encefalopatías o cuadros mentales y que padecen de una depravación del apetito, caen en un consumo reiterado de algunas de sus partes, con las graves consecuencias imaginables.

La más frecuente intoxicación accidental es probablemente la producida por vegetales cianogenéticos. Gran número de plantas son capaces de producir HCN durante su desarrollo, conduciendo casi siempre a cuadros tóxicos de evolución más o menos aguda. Especial mención merecen las Rosáceas frutales, cuyos carozos y pepas representan un peligro potencial para los niños, que los ingieren sobre todo en los huertos y criaderos de frutales. El consumo, a veces masivo, de los carozos y semillas de las diversas especies de cultivo de esta Familia (manzanas, peras, ciruelas, cerezas, melocotones, albaricoques, almendras, etc.) suele plantear un serio problema, al provocar envenenamientos graves e incluso mortales, por perturbación de la respiración celular, la que puede aparecer a veces con muy pequeñas cantidades del tóxico (el fermento respiratorio es transformado en cianferricitocromoxidasa). Los carozos contienen un glucósido cianogenético, la amigdalina, que se desdobla al entrar en contacto con la enzima emulsina, liberándose entonces el ácido cianhídrico. Las fenómenos tóxjcos se producen sobre todo, cuando son ingeridos sin sus vainas o cubiertas envolventes.

Una representatividad menor alcanzan, $\sin d u-$ da, los cuadros tóxicos generados por aspiración y masticación voluntaria, o suministro deliberado a veces con intención suicida u homicida, de un organo vegetal, como asimismo de brebajes preparados con alguna parte constitutiva de una planta.

Uno de ellos, el hábito de fumar hojas triturađas o inflorescencias secas de cánamo, en forma de cigarrillos o cigarros, que tiene claras proyecciones toxicómanas $y$ que se ha difundido con notoria asiduidad, por sus efectos euforísticos, entre nuestros estudiantes secundarios, debe ser considerado como uno de los mecanismos fitotóxicos producidos mediante aspiración buconasal.
Otro tanto puede decirse del vicio tan difundido entre los indios de Perú y Bolivia, de masticar hojas de coca (coqueo), que constituye igualmente una forma de toxicomanía. Esto suele observarse entre los indígenas, tanto arraigados como en tránsito en el extremo norte de nuestro país, inclusive en niños mayores.

Numerosos cocimientos o infusiones de diversos órganos o partes vegetales, algunos de muy reprochables efectos, son aún utilizados en la actualidad, con fines presuntamente curativos. Así p. ej. la costumbre popular de administrar a lactantes una infusión de frutos o cápsulas de adormidera, para tranquilizarlos, ha conducido a graves intoxicaciones.

Merece también ser consignado aquí el procedimiento, un tanto sofisticado, de ingerir hojas de cáñamo conjuntamente con ciertas bebidas, que nuestros adictos juveniles pocas veces suelen utilizar, por la tardía aparición de sus efectos alucinógenos.

Algunas plantas que empleamos como condimentos habituales de nuestra alimentación, contienen ciertas sustancias tóxicas, pero son absolutamente inofensivas en cantidades apropiadas. Cuando son consumidas en exceso, sobre todo por niños pequeños o susceptibles, pueden generar síntomas de envenenamiento. Entre estas especies citaremos la cebolla, el puerro, rábano, rabanito, diversas variedades de coles, el berro, la acedera, la moscatela y la acelga.

Otras plantas comestibles pueden dar lugar a cuadros tóxicos en ciertas fases de su crecimiento, almacenamiento o preparación. Así la humilde e inocente papa, base importante de nuestra dieta, cuando es mal almacenada al final de la estación, al germinar o reverdecer, aumenta su tenor de solanina y puede conducir a envenenamientos muy graves y hasta letales. La ingestión de haba vulgar, al estado verde o bien incompletamente cocida, fermentada o sólo remojada en agua, puede determinar la aparición de un cuadro alergo-tóxico denominado favismo, que puede igualmente revestir mucha gravedad. El garbanzo y la lenteja producen un cuadro similar, conocido como latirismo. Las sopas y cremas de porotos pueden originar intoxicaciones de carácter leve, cuando no han sido suficientemente cocidas (de por sí nuestras variedades de porotos no se prestan para la fabricación de sopas o cremas, por su elevado contenido de $\mathrm{HCN}(47$ a $86 \mathrm{mg} / \mathrm{kg}$.) Los nabos, nutábagas y raíces de remolacha, cuando son mantenidos mucho tiempo en su agua de cocción, pueden también conducir a fenómenos tóxicos, no así cuando se les consume poco después de cocidos. Algunas crucíferas, como coles, repollos, nabos, la rutábaga y coliflor, contienen una sustancia bociógena del grupo de las tiocarbamidas, que cuando es ingerida en cierta proporción por niños ya 
mayores, puede dificultar la síntesis de la hormona tiró́dea y causar en ellos, por un mecanismo de "feed-back" la formación de un bocio (cibario). Los frutos del nogal común (nueces) contienen la misma sustancia, de acción similar.

Plantas contaminadas por causas de tipo ambjental pueden provocar cuadros de muy diversa gravedad.

Así tenemos en primer término aquellos determinados por la ingesta accidental de plaguicidas adheridos a plantas o árboles de cultivo, o accidentalmente a otras plantas o semillas genéticas y que pucden conducir a cuadros graves y a veces mortales.

La espinaca puede producir manifestaciones tóxicas, cuando se le abona en exceso y su contenido de nitrato experimenta una notoria elevación. Al ser guardada a la temperatura ambiente, se observa una abundante formación de nitrito, por acción bacteriana. De este modo puede ocasionar, en determinadas circunstancias, intoxicaciones nitríticas en lactantes pequeños (de 2 a 10 meses), las que regresan espontaneamente o bien después de instituir un tratamiento reductor con azul de metileno. Especialmente en los primeros tres meses de vida no conviene administrar espinaca, porque a esta edad el nitrato puede ser reducido a nivel del intestino delgado y porque existe además una predisposición especial hacia la metahemoglobinemia. Los niños anémicos y con tendencia a padecer trastornos dispépticos son más propensos a este cuadro (9). La ingestión de zanahoria, repollos o coles, que han sido abonados en exceso, puede producir el mismo efecto metahemoglobinizante.

Envenenamientos de carácter leve se pueden generar por consumo de productos de chacarería que han estado expuestos a la acción de emanaciones tóxicas que escapan de las usinas y que se depositan en los cultivos de los campos adyacentes. Entre estas sustancias nocivas las más comunes son el ácido sulfúrico, los fluoruros y el polvo proveniente de las fábricas de cemento.

En algunos sectores industriales, donde se arrojan desperdicios tóxicos a los canales de regadío, también se han observado envenenamientos por consumo de verduras que han sido regadas con estas aguas. Igual cosa ocurre con centros mineros, donde el agua cmpleada cn las plantas concentradoras de cobre o contaminada por residuos de fundiciones de plomo y de otros minerales, es desviada a los cauces naturales.

También diversos alimentos contaminados con sustancias tóxicas de procedencia vegetal, como ser harina, miel y leche y en mucho menor proporcion carnes, conejos, codornices y caracoles, pueden provocar envenenamjentos. Así en nuestro medio se ban presentado con relativa frecuencia intoxicaciones accidentales por consumo de hari- nas contaminadas por semillas tóxicas. (mostaza negra, ricino, chamico, simientes inmaduras de lino (linaza), etc. En 1969 se produjo en la localidad de Nacimiento, provincia de Concepción, una intoxicación masiva de más del $40 \%$ de la población, que incluyó naturalmente a un numeroso contingente infantil, que había consumido pan contaminado con hiosciamina, principio activo del chamico o higuera loca (Datura stramonium L.) (10). El chamico suele crecer en los sembrados de trigo y así fácilmente sus semiłlas pueden ser molidas conjuntamente con el cereal.

El néctar de algunas plantas tóxicas, como azalea, rododendron, acónito, digital y laurel japonés, puede transmitir glucósidos o alcaloides a la miel que con él se elabora y dar así lugar, indirectamente, a intoxicaciones leves, sobre todo en niños pequeños.

Algunas especies, como el tartago o contrarayo, las pichogas o albahaquillas, las tupas $\mathrm{y} \mathrm{cl}$ ricino, transmiten sus propiedades tóxicas a la leche de vaca, provocando cuadros a veces graves $\mathrm{e}$ incluso letales en lactantes.

Los opiáceos pueden pasar también a la leche materna e intoxicar de esa manera al lactante (convicne recordar aquí la gran sensibilidad del niño pequeño a los opiáceos). Igual cosa ocurre con las nodrizas cocainómanas.

La ingestión de carne de cerdos, ovinos o conejos, que consumieron semillas tratadas con ciertos fungicidas, puede originar cuadros tóxicos de gravedad. Entre éstos cabe señalar al provocado por la diciandiamida de mercurio, que se caracteriza por ataxia, perturbaciones de la visión y estado de coma. Corresponde asimismo mencionar aquí un tipo de envenenamiento que se presenta en muy raras ocasiones, en niños que consumieron carne, en cuya preparación se ha utilizado, como asador alguna rama de una especie vegetal tóxica (pillo-pillo) (Ovidia pillo-pillo Meisn.). También han sido descritos casos de intoxicaciones por ingesta de codornices que habían picoteado semillas de vegetales venenosos, inofensivas para ellas (cicuta entre otros) y de caracoles que se habían alimentado de hojas de Deu o Coriaria (Coriaria myrtifolia L.).

Algunos alérgenos que actúan por inbalación (polen de las gramíneas o de flores primaverales, y excepcionalmente también de algunos árboles (tilo, plátano oriental, nogal, saúco, abedul, sauce, álamo)), por contacto con la piel o por ingestión, pueden provocar en ciertos niños un proceso reaccional (fiebre del heno, asma bronquial, urticaria, etc.), cuyo mecanismo de producción (químico, físico o glandular) aún no es bien conocido. Esta reacción polinósica tiene generalmente carácter estacional, al presentarse periódicamente en la época de la floración de determinadas plantas herbáceas o árboles. El hecho que ciertos niños sean suscep- 
tibles a Ia sensibilización alérgica y otros no, parece depender de un factor constitucional, a veces hereditario, aún no bien establecido.

Un capítulo aparte de la toxicología vegetal se refiere a los efectos nocivos que puede ocasionar la medicación herbaria casera, cuyo riesgo potencial es mayor en los niños. Su aplicación no médica, con fines curativos, suele hacerse descontrolada e indiscriminadamente, con desconocimiento tanto de sus propiedades terapéuticas, como de sus inconvenientes. De este modo se pueden fácilmente engendrar cuadros tóxicos secundarios, que plantean dificultades de tipo diagnóstico, que el pediatra muchas veces no logra resolver, dada su complejidad. En todo caso conviene que éste realice un estudio de las medicaciones caseras que son utilizadas en su respectiva área de actividad y las catalogue según su eventual toxicidad. Las que sean inocuas deben ser respetadas; si su uso puede provocar síntomas, según la concentración del producto activo, es importante que se supervise y dirija la técnica de preparación, y por último, deberán contraindicarse aquellas capaces de causar enfermedad. En algunos casos la complejidad del cuadro clínico y la existencia de una patología básica motivante de la práctica, determinan la necesidad de utilizar métodos diagnósticos complementarios que permitan descartar otro tipo de patología (invaginación intestinal, meningitis, etc.).

El cuadro clínico de las intoxicaciones vegetales es con frecuencia vago y a la vez abigarrado, motivo por el cual siempre se debe considerar la posibilidad de su ocurrencia, cuando nos enfrentamos a sindromes de diagnóstico poco claro.

La anamnesis recogida de los familiares, de testigos presenciales o por último, del propio paciente, constituye casi siempre el elemento de juicio más valioso para llegar al diagnóstico etiológico. El ballazgo de las partes vegetales que presumiblemente ban originado la intoxicación, puede ser de decisiva importancia y en tales casos se deberá contar con un buen asesoramiento botánico para efectuar su examen y clasificación.

Atendiendo a su evolución, las intoxicaciones vegetales, al igual que las demás, pueden distinguirse en agudas, de invasión rápida y acción masiva del veneno (hay incluso casos sobreagudos $y$ mortales, entre estos últimos los provocados por el Tejo (Taxus bacatta L.) y diversas especies cianogenéticas), y crónicas, por absorción y acción lenta de pequeñas cantidades, a menudo reiteradas del mismo, que corresponden a cuadros de naturaleza toxicómana (canabismo, tabaquismo, cocaínismo).

Vehiculados por la sangre, los tóxicos circulan por todo el organismo, pudiendo afectar a cualquiera de sus regiones u órganos. Muchos de ellos (en especial alcaloides) son selectivos, porque atacan más intensamente a las células de determi- nados tejidos; otros, al ser tomados con regularidad, en pequeñas dosis y durante un cierto período, tienen efectos acumulativos, debido a que su excreción renal es demasiado lenta y/o su detoxicación hepática insuficiente.

Cabe hacer notar todavía que muchos casos de consecuencias irreparables comienzan con síntomas poco alarmantes y sus posibilidades de recuperación disminuyen a medida que transcurre el tiempo.

Existe un amplio predominio de aquellas plantas cuyos tóxicos son irritantes, que provocan la inflamación de los tejidos con que se ponen en contacto, - piel y mucosas-, y que son absorbidos posteriormente por el torrente sanguíneo. En la primera originan las más diversas lesiones, -dermatitis, eczemas, exantemas, urticarias, ulceraciones y gangrena--, y a niveI de las segundas, -conjuntivas, fosas nasales, cavidad bucal, estómago e intestino-, una gama muy versátil de manifestaciones inflamatorias.

Entre las plantas que provocan vesicación e inflamación cutánea, se debe señalar especialmente el litre, chamico, quillay, cornezuelo de centeno, la clemátida y la centella. El zumo acuoso o lechoso (látex) de algunas especies puede originar lesiones de carácter ulceroso; otras, como las ortigas, cuyas hojas y tallos están cubiertos de pelos especiales (urticantes), producen un efecto irritante de variada intensidad y por último, determinadas plantas son tóxicas por un mecanismo alérgico. Basta tocarlas o detenerse bajo su follaje, para que aparezcan erupciones y vesículas, a veces muy dolorosas o intensamente pruriginosas.

Especialmente llamativa es la elevada proporción de los trastornos gastrointestinales, ya que éstos se presentan en la mayoría de los envenenamientos.

El sindrome más completo está constituido por: sensación bucal especial, sialorrea, náuseas, vómitos, dolores en el segmento superior del tubo digestivo, dolores abdominales y diarreas.

La sensación bucal es en algunos casos gustativa (sabor amargo, dulzón o sui géneris) y en otros dolorosa, desde un ardor más o menos fuerte, hasta un dolor franco, localizándose en la mucosa bucal, en la lengua, las fauces y la faringe. La sialorrea es especialmente notoria en los cuadros provocados por el acónito, aro común, cicuta, contrahierba, coriaria estafisagria, hierba mora, hino. jo, huayo, ruda y tabaco.

Los vómitos aparecen después de un tiempo variable y pueden ser debidos a la acción central del veneno, como también a la irritación de la mucosa gástrica. Grandes dosis del tóxico generalmente los aceleran e intensifican. En determinadas intoxicaciones, los vómitos tienen algunas características especiales que orientan el diagnóstico. Así por ej. la marihuana les confiere un color verde, 
algunas especies un olor aliácco, el HCN uno a esencia de almendras.

Cuando el veneno llega al intestino y es cáustico para la mucosa del mismo, sobreviene la últjma parte del sindrome, compuesta esencialmente por los cólicos y la diarrea. Los cólicos son generailmente dolores abdominales breves y agudos. A veces, sin embargo, el dolor abdominal es difuso y sin carácter espasmódico. La diarrea habitualmente es debidia a una irritación directa de las paredes intestinales, que a veces puede ser bastante violenta (ricina, colchicina); sólo rara vez tiene relación con sustancias que actúan sobre el sistema nervioso. Se la debe considerar como un medio natural para expulsar prontamente las sustancias irritantes del intestino, por 10 que no conviene adoptar ringuna medida para conjurarla en las primeras fases de la intoxicación. La ingestión excesiva de especies inocuas puede prestarse a una interpretación equivocada del cuadro, al generar también una grave irritación o inflamación gastrointestinal.

Cabe senalar igualmente el frecuente compromiso de los sistemas nervioso central y ncuromuscular; la mayor parte de los cuadros lo evidencian en algún momento de su evolución y sus síntomas siguen en frecuencia a los gastroentéricos. Algunos venenos vegetales causan estos trastornos de un modo exclusivo o preponderante, otros lo hacen sólo accesoriamente; pero siempre hay una repercusión del proceso tóxico sobre los órgnos nerviosos. Dicha repercusión se puede manifestar funcionalmente por una excitación o por una depresión del sistema. Especialmente los cuadros tóxicos producidos por chamico, tabaco, cólchico, acónico, adormidera y digital, se caracterizan por síntomas reveladores de un compromiso nervioso bien definido. Por parte del sistema neuromuscular merecen consignarse especialmente las contracturas y los temblores musculares. Por últi. mo, algunos tóxicos pueden modificar la temperatura, por diferentes mecanismos, al actuar sobre los centros termoreguladores. Así el chamico, la coca y el quillay pueden producir hipertermia; el acónito y la adormidera, en cambio, un estado de hipotermia.

De reiterada observación en las intoxicaciones por vegetales son también los signos cardiovasculares, sobre todo alteraciones del pulso (número de pulsaciones y ritmo) y de la presión arterial (hiper o hipotensión).

Los venenos inhalados (tabaco, marihuana, pólenes de ciertas plantas herbáceas y árboles, etc.) producen diversas lesiones o cuadros respiratorios, cuya magnitud depende del grado de toxicidad y del tiempo de acción de la sustancia absorbida. Entre estas lesiones hay que mencionar estados catarrales nasofaringeos y laríngeos, o cuadros de edema pulmonar, en las intoxicaciones agu- das, y catarros localizados en la mucosa traqueal y bronquial, en ciertos casos crónicos. La exaltación de lá virulencia de los gérmenes, puede en algunos envenenados conducir, posteriormente, a procesos neumónicos, bronconeumónicos o pleurales.

El compromiso renal puede revistir variadas características. Así algunas toxoalbúminas como la ricina provocan una lesión del glomérulo; otras sustancias venenosas, como las de algunos hongos, conducen a lesiones tubulares y por último, una tercera posibilidad, consiste en la formación de precipitados (cristales de oxalato de calcio p. ej.) dentro de los túbules (vinagrillo, rimu, acederas). No se ha observado cuadros crónicos caracterizados por lesiones del tejido intersticial y proliferación conjuntiva.

Por su condición de importante órgano antitóxico, el hígado puede sufrir lesiones más a menos serias, por la acción de diversas sustancias agresivas. Así, entre los clásicos venenos hepáticos se cuenta la toxoalbúmiria de algunos hongos; la hualtata y el ricino pueden provocar una necrosis o degeneración grasa del órgano y diversas otras especies, como la haba vulgar y el paico, pueden determinar también un frecuente daño del mismo.

Algunas intoxicaciones producen alteraciones sanguíneas, como ser aumento de la coagulabilidad (ricina), hemolisis (favismo), metahemoglobinemia (espinaca), cianohemoglobinemia (rosáceas frutales), hipocalceraia (vinagrillo, berro), pero su número no es significativo.

En un mismo intoxicado los síntomas se pueden combinar de manera imprevisible, determinando distintas formas clínicas. Asimismo, en las diversas fases o etapas de una misma intoxicación, se puede producir el llamado "antagonismo sintomático", como ser excitación/sopor, bradicardia/ taquicardia.

En cuanto al tratamiento de los envenenamientos vegetales, éste sigue los lineamientos generales de la terapéutica toxicológica. Debe estar siempre dirigido a la solución de cada una de las situaciones presentes: deshidratación, excitación, coma, acidosis, convulsiones, temblores, fiebre, edema de glotis $\mathrm{y} / \mathrm{o}$ pulmonar, parálisis respiratoria, etc.

En el medio rural, algunas especies ban sido utilizadas como contraveneno, en determinados cuadros tóxicos: hierba mora (Solanum nigrum L.) en intoxicaciones por latué (Latua pubiflora Griseb), ambas Solanáceas; el palqui (Cestrum parqui L'Hérit), solanácea, en el tratamiento de los accidentes provocados por el litre (Lithraea cáustica Miers), Amacardiácea. La utilidad de su empleo no ha sido aún confirmada en definitiva. Cabe señalar, por último, algunas prácticas profilácticas, de aparente eficacia, que el empirismo y la medicina popular han preconizado igualmente en sectores rurales. Entre éstas puede consignarse 
la ingestión de semillas de litre para prevenir la aparición de las típicas manifestaciones alergotóxicas que puede ccasionar este árbol.

\section{REFERENCIAS}

1.- Bruning, W.; Orrego, M.; Saglietto, G. y Becker, $M$. Morbilidad toxicológica en Chile. Rev. Chilena de Pediatría. № 1, 1968.

2.-Astolfi, E.; Banzas, T. y Giménez, E. Labor del Centro de Informaciones Toxicológicas del Hospital de Niños de Bnenos Aires. Rev. Asoc. Méd. Arg. Vol. $77, \mathrm{~N}^{\circ} 11,1963$.

3.-Moeschlin, Sv. Klinik und Therapie der Vergiftungen. 4. Auflage. Thieme Stuttgart, 1964.
4.- Schvarisman, $S$. Información sobre la situación toxicológica de Brasil. Comunicación al 29 Congreso L. A. de Toxicología, 1971.

5.-Bruning, W, y cols. Citado en (1).

6.-Wirth, W.i Hechi, G. y Gloxhuler, Ch. Toxikologie Fibel. 2. Auflage. Thieme Stuttgart, 1971.

7.-Schvartsman, S. Citado en (4).

8.- National Clearinghouse for Poison Control Centers. Washington. Información estadística periódica. 1967.

9.-Simon, C. Nitritvergiftungen beì Säuglingen nach Spinatgenuss. Med. u. Ernähr. 7, 262, 1966.

10.-Bruning, W. Plantas tóxicas de América Latina (se publicará en breve). 